\title{
Review
}

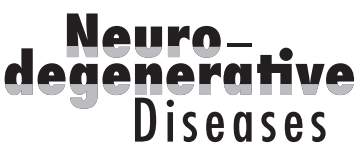

\section{Tau Immunotherapy}

\author{
Einar M. Sigurdsson \\ Departments of Neuroscience and Physiology, and Psychiatry, New York University School of Medicine, \\ New York, N.Y., USA
}

\section{Key Words}

Tau immunotherapy - Tauopathies · Alzheimer's disease

\begin{abstract}
In recent years, tau immunotherapy has advanced from proof-of-concept studies [Sigurdsson EM, NIHR01AG020197, 2001; Asuni AA, et al: J Neurosci 2007;27:9115-9129], which have now been confirmed and extended by us and others. Phase I clinical trials on active and passive tau immunizations are being conducted, with several additional passive tau antibody trials likely to be initiated in the near future for Alzheimer's disease and other tauopathies. Because tau pathology correlates better with the degree of dementia than amyloid- $\beta(A \beta)$ pathology, greater clinical efficacy may be achieved by clearing tau than $A \beta$ aggregates in the later stages of the disease, when cognitive impairments become evident. Substantial insight has now been obtained regarding which epitopes to target, mechanism of action and potential toxicity, but much remains to be clarified. All of these factors likely depend on the model/disease or stage of pathology and the immunogen/antibody. Interestingly, tau antibodies interact with the protein both extra- and intracellularly, but the importance of each site for tau clearance is not well defined. Some antibodies are readily taken up into neurons, whereas others are not. It can be argued that extracellular clearance may be safer but less efficacious than in-
\end{abstract}

traneuronal clearance and/or sequestration to prevent secretion and further spread of tau pathology. Development of therapeutic tau antibodies has led to antibody-derived imaging probes, which are more specific than the dye-based compounds that are already in clinical trials. Such specificity may give valuable information on the pathological tau epitope profile, which could then guide the selection of therapeutic antibodies for maximal efficacy and safety. Hopefully, tau immunotherapy will be effective in clinical trials, and further advanced by mechanistic clarification in experimental models with insights from biomarkers and postmortem analyses of clinical subjects.

(c) 2015 S. Karger AG, Basel

Tau immunotherapies are now moving into clinical trials, but the field is still in its infancy, and mechanistic understanding of the efficacy and safety of the various approaches is not well established. It is noteworthy that we do not fully understand the mechanism of action of amyloid- $\beta(A \beta)$ antibodies after 16 years of work by numerous groups or for that matter $A \beta$ biology after 30 years of studies. With less research into tau biology over the years and multiple more epitopes to target, because of its size and posttranslational modifications, we have a long way to go to clarify the mechanisms involved in tau immunotherapies.

\section{KARGER}

E-Mail karger@karger.com

www.karger.com/ndd
(C) 2015 S. Karger AG, Basel

$1660-2854 / 15 / 0162-0034 \$ 39.50 / 0$
Dr. Einar M. Sigurdsson

Department of Neuroscience and Physiology, New York University School of Medicine 550 First Avenue, Medical Science Building, MSB459

New York, NY 10016 (USA)

E-Mail einar.sigurdsson@ nyumc.org 


\section{Which Epitopes to Target}

Some insights have been obtained regarding which epitopes may be best to target, although other properties of antibodies such as affinity, charge and isotype are likely to be important as well. The epitope that has received the most attention, phosphoserine 396/404 which was the key part of the immunogen in the original report [1], has now been confirmed to be a feasible target in several studies [2-11], and such a vaccine is being employed in one of the ongoing clinical trials $[6,12]$. A few studies have compared the efficacy of antibodies binding to different regions of the tau protein $[4,7,9-11,13,14]$, providing additional clarification although the differences observed may not only be epitope dependent but can be influenced by other properties of the antibodies such as affinity, charge and isotype. Other obvious variables that apply when comparing different studies include: the model, age of animals, tau protein expressed and expression levels, dose, number and route of injections, and adjuvant used for active immunizations.

It may be preferable to compare target engagement and pharmacokinetics of antibodies against key epitopes as well as immune responses and similar measures of certain active immunogens in phase I human trials instead of seeking direct comparison in large animal studies. Beside well-known differences in immune systems, which can influence efficacy and safety, humanized antibodies are not exactly the same as the original mouse monoclonal antibodies. Relatively subtle differences in charge, effector function and binding specificity as well as target differences may substantially alter the outcome. For detailed insight into ongoing tau immunotherapy programs that are actively seeking a clinical candidate, see a recent review [12]. Six phase I trials are currently in progress. Two of those are active and 4 are passive immunotherapies. The first trial that was started by Axon Neuroscience SE is on an active immunization approach using a tau fragment, tau294-305, linked to keyhole limpet hemocyanin through an $\mathrm{N}$-terminal cysteine and administered with an alum adjuvant. It was reportedly designed to target misfolded tau, and its safety is being assessed in patients with mild-to-moderate Alzheimer's disease [15, 16]. This particular epitope has not been pursued by others at this point. The second active trial by AC Immune and Janssen employs the phosphoserine 396/404 epitope in a liposome adjuvant [6], based on the company's website, but other information on this trial has not been released. It is not clear if it is being conducted in tauopathy patients or healthy subjects. Two of the passive trials are

Tau Immunotherapy only enrolling healthy individuals, and 2 are focusing on subjects with progressive supranuclear palsy. Two of these are being performed by Bristol-Meyers Squibb on one of the tau antibodies they obtained with their purchase of iPerian $[17,18]$. It targets a postulated pathological tau fragment (tau1-224) but should react with total normal tau as well [19]. Hence, the trials may clarify if there are any safety issues with targeting normal tau in healthy subjects, as well as both normal and pathological tau in progressive supranuclear palsy subjects. If animal data is any indication [1], fewer of these antibodies can be expected to get into the brain of normal individuals compared to tauopathy subjects who are likely to have a leaky blood-brain barrier. If everything goes well, this antibody will presumably be tested in Alzheimer's patients and other tauopathies. Another passive immunotherapy trial is being conducted by Roche on their antibody against phosphoserine 422 [20]. This trial involves healthy subjects who should not have this epitope as it is thought to be only found in tauopathies [21]. Hence, studying target engagement is likely not one of the objectives, but this initial trial could confirm that the epitope is indeed specific to tau pathology as no changes should be expected in tau levels in plasma or cerebrospinal fluid in these subjects. As with the Bristol-Meyers Squibb trial, this antibody will likely be tested soon in one or more tauopathies. A third antibody, C2N-8E12, developed by C2N Diagnostics, recently entered phase I in progressive supranuclear palsy patients, in collaboration with AbbVie [22]. It is not clear if this is the humanized version of the apparent lead antibody of C2N Diagnostics, HJ8.5 [14], which recognizes an $\mathrm{N}$-terminal epitope (amino acids 25-30) found in its normal and pathological forms.

As mentioned above, several other approaches are in late-stage preclinical development and likely to enter clinical trials in the near future [for a review, see 12]. Many other promising tau immunotherapies are actively being studied, but it is not yet clear if those will pursue clinical trials [for reviews, see 12 and 23].

\section{Mechanism of Action}

In theory, the tau antibodies can interact with their target both extra- and intracellularly. It is conceivable that extracellular clearance may be safer but less efficacious than intraneuronal clearance and/or sequestration to prevent secretion and further spread of tau pathology. Preliminary findings from this ADPD 2015 conference support the efficacy argument, showing improved life expec- 
tancy in tangle mice that express intracellularly a tau antibody fragment, compared to an identical fragment that was directed into the secretory pathway [24]. The importance of each pathway likely depends on the availability of the epitope being targeted within each compartment as well as the properties of the antibodies, mainly their charge which influences their entry into cells. It is well established in other immunotherapy fields that manipulating the isoelectric point of antibodies with bioengineering can greatly influence their cell permeability [25]. Generally speaking, acidic antibodies should have lower cell penetrance. The tau antibodies that we have generated and studied in some detail are easily taken up into neurons in various culture and in vivo assays as assessed by different techniques. These antibodies have an isoelectric point at or near the neutral range ( $\mathrm{pH}$ 6-8). In all likelihood, tau antibodies that have been reported not to be taken up into cells/neurons are acidic. Such antibodies would be negatively charged and be repelled from the negatively charged cell surface. For this reason, their half-life may be longer and therefore to some extent make up for their limited access to the intraneuronal pool of tau aggregates.

Within these different compartments, several different clearance pathways are possible. Extracellularly, the most prominent scenario is likely microglial phagocytosis of the antibody-tau complex. Intracellularly, the majority of the clearance is presumably via the endosomal/lysosomal pathway, in which tau antibodies have been detected complexed to tau aggregates. This binding may disassemble the tau aggregates and thereby allow better access of lysosomal enzymes to degrade those assemblies.

\section{Other Considerations}

It is not clear if antibodies with the highest affinities are necessarily the best ones to clear tau pathology, and this may depend on the epitope and if they are acting intraand/or extracellularly. Very strong binding to particular epitopes may prevent degradation of tau assemblies and/ or promote aggregation which could then seed further aggregation and be detrimental. High affinity could also reduce the half-life of the antibody as the bound antibody could not be reused. On the other hand, formation of large antibody-tau complexes could be helpful up to a point by sequestering smaller aggregates and thereby slow the spread/progression of the disease. It is conceivable that relatively low-affinity antibodies that still maintain a high degree of specificity may be preferable as therapeutic antibodies by tilting these diverse pathways towards degra- dation instead of furthering aggregation. Lower-affinity antibody may come off the tau molecule with $\mathrm{pH}$ changes in endosomes and be exocytosed and could therefore interact with other tau molecules. This could result in a longer half-life of the antibody and a lower dose for efficacy. Such examples exist from other fields [25]. Ideally, this needs to be explored with antibodies against the same epitope that only differ in their affinities towards it. However, hints of this possibility have been seen with tau antibodies against different epitopes with low-affinity antibody towards a conformational epitope showing efficacy in clearing tau pathology, whereas high-affinity antibody against total tau was not effective in the same mouse model [13]. Targeting both normal and pathological tau may also reduce efficacy as binding to normal tau will sequester the antibody, which reduces the amount of antibodies that can bind to the pathological form.

There are some indications that isotype differences can influence antibody-mediated degradation. One report has suggested that this may be the case with two antibodies of similar affinity against the same epitope showing isotype-dependent efficacy [7]. This interesting finding needs to be confirmed with antibodies that are identical except for their isotype, and any observed differences in efficacy clarified mechanistically.

\section{Toxicity}

Rosenmann et al. [26] have examined the potential toxicity of immunization with full-length recombinant tau administered with a combination of very strong adjuvants that promote cytotoxic $\mathrm{T}$-cell response. This represents the worst-case scenario as full-length tau is likely to have some detrimental T-cell epitopes that may promote autoimmunity, and their influence is then exacerbated with the combination of the very robust adjuvants that would not be approved for human use. As outlined in the publication, the study was undertaken to test the feasibility of an active induction of a neuroautoimmune disorder in mice. The immunized wild-type mice developed tauopathy-like abnormalities and neurological deficits. More recently, no side effects were reported by another group using a different model of tauopathy mice that were immunized with a similar full-length tau but administered with a milder adjuvant [27]. Rosenmann's group subsequently showed that repeated immunizations with phospho-tau peptides emulsified in the same strong adjuvants had detrimental effects in both tauopathy and wild-type mice confirming that such adjuvants are too strong to be
Sigurdsson 
used when self-epitopes are being targeted [28]. Likewise, we have observed mouse death under certain conditions in some animal models which appears to be related to a strong immune response to at least some tau immunogens that may be background strain related [unpubl. observations]. Most recently, enhanced mortality was observed in $A \beta$ plaque mice immunized with a total tau antibody, suggesting that it may be safer to target pathological tau epitopes [29], although that should be studied further in the same model. As detailed above, certain total tau antibodies have successfully cleared tau pathology without reported side effects in models that overexpress the tau protein $[14,19]$. Although it is not particularly likely that such antibodies will come into extensive contact with normal tau as discussed previously $[1,30]$, this may depend on their properties. In light of the death induced by a particular total tau antibody in the $A \beta$ plaque model, this potential issue should be explored further in various models with normal tau levels.

\section{Imaging Studies}

At least 5 small-molecule $\beta$-sheet dye-derived positron emission tomography imaging ligands have shown promise in pilot clinical studies [31-35]. It is likely that these will be used in some of the ongoing and upcoming clinical trials on tau immunotherapies to verify the presence of tauopathy and to monitor treatment efficacy. However, antibody derivatives that have shown promise in animal models should be more specific for tau pathology than these $\beta$-sheet binders that inevitably will have some affinity for other amyloids [36]. Furthermore, such antibodybased probes may allow detailed information on the pathological tau epitope profile of each imaged subject which could then direct personalized immunotherapy strategy targeting those specific epitopes.

\section{Concluding Remarks}

As this brief overview indicates, these are exciting times for tau immunotherapies, but much remains to be done to advance this field into approved therapies. Ongoing mechanistic studies should nicely complement the clinical trials and should provide important insights for further development of this promising approach.

\section{Acknowledgments}

E.M.S. is supported by NIH grants NS077239, AG032611 and AG020197. He is an inventor of patents on tau immunotherapy and related diagnostics that are assigned to New York University. This technology is licensed to and is being codeveloped with $\mathrm{H}$. Lundbeck A/S.

\section{Disclosure Statement}

The author has no conflict of interest to declare.

\section{References}

$>1$ Asuni AA, Boutajangout A, Quartermain D, Sigurdsson EM: Immunotherapy targeting pathological tau conformers in a tangle mouse model reduces brain pathology with associated functional improvements. J Neurosci 2007;27:9115-9129.

2 Boutajangout A, Quartermain D, Sigurdsson EM: Immunotherapy targeting pathological tau prevents cognitive decline in a new tangle mouse model. J Neurosci 2010;30:1655916566.

-3 Boutajangout A, Ingadottir J, Davies P, Sigurdsson EM: Passive immunization targeting pathological phospho-tau protein in a mouse model reduces functional decline and clears tau aggregates from the brain. J Neurochem 2011;118:658-667.
4 Chai X, Wu S, Murray TK, Kinley R, Cella CV, Sims H, Buckner N, Hanmer J, Davies P, O’Neill MJ, Hutton ML, Citron M: Passive immunization with anti-tau antibodies in two transgenic models: reduction of tau pathology and delay of disease progression. J Biol Chem 2011;286:34457-34467.

5 Bi M, Ittner A, Ke YD, Gotz J, Ittner LM: Tautargeted immunization impedes progression of neurofibrillary histopathology in aged P301L tau transgenic mice. PLoS One 2011; 6:e26860.

6 Theunis C, Crespo-Biel N, Gafner V, Pihlgren M, Lopez-Deber MP, Reis P, Hickman DT, Adolfsson $\mathrm{O}$, Chuard N, Ndao DM, Borghgraef P, Devijver H, van Leuven F, Pfeifer A, Muhs A: Efficacy and safety of a liposome-based vaccine against protein tau, assessed in tau.P301L mice that model tauopathy. PLoS One 2013;8:e72301.
Ittner A, Bertz J, Suh LS, Stevens CH, Gotz J, Ittner LM: Tau-targeting passive immunization modulates aspects of pathology in tau transgenic mice. J Neurochem 2015;132:135-145.

-8 Congdon EE, Gu J, Sait HB, Sigurdsson EM: Antibody uptake into neurons occurs primarily via clathrin-dependent Fcgamma receptor endocytosis and is a prerequisite for acute tau protein clearance. J Biol Chem 2013;288: 35452-35465.

$9 \mathrm{Gu}$ J, Congdon EE, Sigurdsson EM: Two novel tau antibodies targeting the 396/404 region are primarily taken up by neurons and reduce tau protein pathology. J Biol Chem 2013;288: 33081-33095.

10 Umeda T, Eguchi H, Kunori Y, Matsumoto Y, Taniguchi T, Mori H, Tomiyama T: Passive immunotherapy of tauopathy targeting pSer413-tau: a pilot study in mice. Ann Clin Transl Neurol 2015;2:241-255. 
11 Sankaranarayanan S, Barten DM, Vana L, Devidze N, Yang L, Cadelina G, Hoque N, DeCarr L, Keenan S, Lin A, Cao Y, Snyder B, Zhang B, Nitla M, Hirschfeld G, Barrezueta N, Polson C, Wes P, Rangan VS, Cacace A, Albright CF, Meredith J Jr, Trojanowski JQ, Lee VM, Brunden KR, Ahlijanian M: Passive immunization with phospho-tau antibodies reduces tau pathology and functional deficits in two distinct mouse tauopathy models. PLoS One 2015;10:e0125614.

12 Pedersen JT, Sigurdsson EM: Tau immunotherapy for Alzheimer's disease. Trends Mol Med 2015;21:394-402.

13 D'Abramo C, Acker CM, Jimenez HT, Davies P: Tau passive immunotherapy in mutant P301L mice: antibody affinity versus specificity. PLoS One 2013;8:e62402.

14 Yanamandra K, Kfoury N, Jiang H, Mahan TE, Ma S, Maloney SE, Wozniak DF, Diamond MI, Holtzman DM: Anti-tau antibodies that block tau aggregate seeding in vitro markedly decrease pathology and improve cognition in vivo. Neuron 2013;80:402-414.

15 Kontsekova E, Zilka N, Kovacech B, Novak P, Novak M: First-in-man tau vaccine targeting structural determinants essential for pathological tau-tau interaction reduces tau oligomerisation and neurofibrillary degeneration in an Alzheimer's disease model. Alzheimers Res Ther 2014;6:44.

16 Axon Neuroscience SE: NCT01850238: safety study of AADvac1, a tau peptide-KLH-conjugate active vaccine to treat Alzheimer's disease. 2015. https://ClinicalTrials.gov.

17 Bristol-Meyers Squibb: NCT02294851: a randomized, double-blind, placebo-controlled, single ascending dose study of intravenously administered BMS-986168 in healthy subjects. 2015. https://ClinicalTrials.gov.

18 Bristol-Meyers Squibb: NCT02460094: multiple ascending dose study of intravenously administered BMS-986168 in patients with progressive supranuclear palsy (CN002-003). 2015. https://ClinicalTrials.gov.

19 Bright J, Hussain S, Dang V, Wright S, Cooper B, Byun T, Ramos C, Singh A, Parry G, Stagliano N, Griswold-Prenner I: Human secreted tau increases amyloid-beta production. Neurobiol Aging 2015;36:693-709.
20 Hoffmann-La-Roche: NCT02281786: a study of RO6926496 in healthy volunteers. 2015. https://ClinicalTrials.gov.

21 Troquier L, Caillierez M, Burnouf S, Fernandez-Gomez FJ, Grosjean MJ, Zommer N, Sergeant N, Schraen-Maschke S, Blum D, Buee L: Targeting phospho-Ser422 by active tau immunotherapy in the THY-Tau22 mouse model: a suitable therapeutic approach. Curr Alzheimer Res 2012;9:397-405.

22 C2N Diagnostics - Abbvie: NCT02494024: safety, tolerability and pharmacokinetics of C2N-8E12 in subjects with progressive supranuclear palsy. 2015. https://ClinicalTrials.gov.

23 Congdon EE, Krishnaswamy S, Sigurdsson EM: Harnessing the immune system for treatment and detection of tau pathology. $\mathrm{J} \mathrm{Alz}$ heimers Dis 2014;40(suppl 1):S113-S121.

24 Levites Y, Sinyavskaya O, Rosario AM, Cruz $\mathrm{PE}$, Lewis J, Golde TE: Targeting intra- vs extracellular tau by recombinant antibodies. Neurodegener Dis 2015;15:337.

25 Igawa T, Tsunoda H, Kuramochi T, Sampei Z, Ishii S, Hattori K: Engineering the variable region of therapeutic IgG antibodies. MAbs 2011;3:243-252.

26 Rosenmann H, Grigoriadis N, Karussis D, Boimel M, Touloumi O, Ovadia H, Abramsky O: Tauopathy-like abnormalities and neurologic deficits in mice immunized with neuronal tau protein. Arch Neurol 2006;63:14591467.

27 Selenica ML, Davtyan H, Housley SB, Blair LJ, Gillies A, Nordhues BA, Zhang B, Liu J, Gestwicki JE, Lee DC, Gordon MN, Morgan D, Dickey CA: Epitope analysis following active immunization with tau proteins reveals immunogens implicated in tau pathogenesis. J Neuroinflamm 2014;11:152.

28 Rozenstein-Tsalkovich L, Grigoriadis N, Lourbopoulos A, Nousiopoulou E, Kassis I, Abramsky O, Karussis D, Rosenmann H: Repeated immunization of mice with phosphorylated-tau peptides causes neuroinflammation. Exp Neurol 2013;248:451-456.

29 Mably AJ, Kanmert D, McDonald JM, Liu W, Caldarone BJ, Lemere CA, O'Nuallain B, Kosik KS, Walsh DM: Tau immunization: a cautionary tale? Neurobiol Aging 2015;36: 1316-1332.
30 Sigurdsson EM: Immunotherapy targeting pathological tau protein in Alzheimer's disease and related tauopathies. J Alzheimers Dis 2008;15:157-168.

- 31 Maruyama M, Shimada H, Suhara T, Shinotoh H, Ji B, Maeda J, Zhang MR, Trojanowski JQ, Lee VM, Ono M, Masamoto K, Takano H, Sahara N, Iwata N, Okamura N, Furumoto S, Kudo Y, Chang Q, Saido TC, Takashima A, Lewis J, Jang MK, Aoki I, Ito $\mathrm{H}$, Higuchi M: Imaging of tau pathology in a tauopathy mouse model and in Alzheimer patients compared to normal controls. Neuron 2013;79:1094-1108.

32 Chien DT, Bahri S, Szardenings AK, Walsh JC, Mu F, Su MY, Shankle WR, Elizarov A, Kolb HC: Early clinical PET imaging results with the novel PHF-tau radioligand [F-18]T807. J Alzheimers Dis 2013;34:457-468.

33 Chien DT, Szardenings AK, Bahri S, Walsh JC, Mu F, Xia C, Shankle WR, Lerner AJ, Su MY, Elizarov A, Kolb HC: Early clinical PET imaging results with the novel PHF-tau radioligand [F18]-T808. J Alzheimers Dis 2014;38: 171-184.

34 Okamura N, Furumoto S, Fodero-Tavoletti MT, Mulligan RS, Harada R, Yates P, Pejoska S, Kudo Y, Masters CL, Yanai K, Rowe CC, Villemagne VL: Non-invasive assessment of Alzheimer's disease neurofibrillary pathology using 18F-THK5105 PET. Brain 2014;137: $1762-1771$.

35 Harada R, Okamura N, Furumoto S, Furukawa K, Ishiki A, Tomita N, Hiraoka K, Watanuki S, Shidahara M, Miyake M, Ishikawa Y, Matsuda R, Inami A, Yoshikawa T, Tago T, Funaki Y, Iwata R, Tashiro M, Yanai K, Arai H, Kudo Y: [(18)F]THK-5117 PET for assessing neurofibrillary pathology in Alzheimer's disease. Eur J Nucl Med Mol Imaging 2015;42: 1052-1061.

36 Krishnaswamy S, Lin Y, Rajamohamedsait WJ, Rajamohamedsait HB, Krishnamurthy P, Sigurdsson EM: Antibody-derived in vivo imaging of tau pathology. J Neurosci 2014;34: 16835-16850. 